\title{
Impact of Travel Distance on Radiation Treatment Modality for Central Nervous System Disease
}

\author{
Shearwood McClelland III,2 Timur Mitin ${ }^{1}$ Jerry J. Jaboin² Jeremy N. Ciporen ${ }^{3}$ \\ ${ }^{1}$ Department of Radiation Oncology, Indiana University School of \\ Medicine, Indianapolis, Indiana, United States \\ 2Department of Radiation Medicine, Oregon Health and Science \\ University, Portland, Oregon, United States \\ ${ }^{3}$ Department of Neurological Surgery, Oregon Health and Science

\begin{abstract}
Address for correspondence Shearwood McClelland III, MD, Department of Radiation Medicine, Indiana University School of Medicine, 535 Barnhill Drive, RT 041, Indianapolis, IN 46202, United States (e-mail: drwood@post.harvard.edu).
\end{abstract} University, Portland, Oregon, United States

J Neurosci Rural Pract 2019;10:606-607

\begin{abstract}
Background Stereotactic body radiation therapy (SBRT) has emerged as a popular alternative to conventional radiation therapy (RT) over the past 15 years. Unfortunately, the impact of patient distance from radiation treatment centers and utilization of SBRT versus conventional RT has been sparsely investigated. This report represents the first analysis of the impact of patient distance on radiation treatment modality for central nervous system (CNS) disease.

Materials and Methods Since the inception of our RADlation oncology And NeuroSurgery (RADIANS) multidisciplinary clinic at a community hospital in 2016, 27 patients have received either SBRT or conventional RT as their sole radiation treatment modality for CNS disease. Twenty-four (88.9\%) presented with metastatic disease. Fisher's exact test evaluated the relationship between patient residence from treatment (in miles) and radiation treatment modality received.

Keywords

- central nervous system metastases

- community hospital

- neurosurgery

- radiation oncology

- radiation oncology and neurosurgery

- stereotactic body radiation therapy

- travel distance

Results Mean patient distance from our RADIANS clinic was 50.6 miles (median $=15.3)$. Twenty-one patients $(77.8 \%)$ received SBRT; the remaining six received conventional RT. Mean patient distance from SBRT was 63.6 miles, and mean patient distance for conventional RT was 5.1 miles; this finding was statistically significant $(p=0.0433 ; 95 \%$ confidence interval $=1.9-115.1)$.

Conclusion Our findings indicate that patients with CNS disease who receive SBRT over conventional RT are statistically more likely to reside further from treatment centers. This is similar to findings of national studies comparing proton versus photon treatment for pediatric solid malignancies. The results from our work have implications for neuro-oncology treatment and the development of community hospital-based clinic models similar to RADIANS in the future.
\end{abstract}

\section{Introduction}

The increasing popularity of stereotactic body radiation therapy (SBRT) as an alternative to conventional radiation therapy (RT) has permeated oncology over the past 15 years..$^{1-3}$ Unfortunately, the impact of patient distance from radiation treatment centers and utilization of SBRT versus conventional RT has been sparsely investigated; a recent report investigating localized prostate cancer found that patients residing more than 25 miles from treatment centers were significantly more likely to receive SBRT than conventional fractionation. ${ }^{3}$ For patients receiving treatment in a community hospital setting, this distance may play a significant role in treatment; many patients reside more than 50 miles away from their nearest treatment center, with some residing more than 250 miles away. This report represents the first 
analysis of the impact of patient distance on radiation treatment modality for central nervous system (CNS) disease.

\section{Materials and Methods}

Since the inception of our RADIation oncology And NeuroSurgery (RADIANS) multidisciplinary clinic at a community hospital in 2016, 27 patients have received either SBRT or conventional RT as their sole radiation treatment modality for CNS disease; the radiation treatment center is located in the same building as the clinic. ${ }^{4}$ Twenty-four of these patients (88.9\%) presented with metastatic disease of the brain or spine. Of these 24 patients, 12 had brain metastases, 11 had spine metastases, and 1 had both brain and spine metastases. Statistical analyses were performed using $t$-tests to evaluate the relationship between patient residence from treatment (in miles), and radiation treatment modality received, with statistical significance assigned at $p<0.05$ (GraphPad Software, San Diego, California, United States).

\section{Results}

Mean patient age was 64.4 (median $=67$ ). Mean patient distance from our RADIANS clinic was 50.6 miles ( median $=15.3$ ). Fifteen patients resided $<25$ miles away from clinic; eight were 51 to 100 miles away, two were 101 to 200 miles away, and two were 200+ miles away from treatment. Twenty-one of the 27 patients received SBRT (77.8\%); the remaining six received conventional RT. Mean patient distance from SBRT was 63.6 miles (standard error of mean $[$ SEM] $=14.5$ ), while for conventional RT mean patient distance from treatment was 5.1 miles ( $\mathrm{SEM}=1.9$ ); this finding was statistically significant $(p=0.0433 ; 95 \%$ confidence interval $=1.9-115.1)$.

\section{Conclusion}

As recently reported for localized prostate cancer, ${ }^{1}$ our findings indicate that patients with CNS disease who receive SBRT over conventional RT are statistically more likely to reside further from treatment centers. This is similar to findings of national studies comparing proton versus photon treatment for pediatric solid malignancies, where patients receiving proton therapy were significantly more likely to travel $>200$ miles to do so. ${ }^{5}$ Unlike for prostate and breast cancer, for CNS disease there is not Level I evidence establishing hypofractionation as equivalent to conventional RT; it is more likely that the paucity of SBRT access in the treatment of brain/spine disease is the catalyst for our distance-related findings. ${ }^{6,7}$ The results from our work have implications for neuro-oncology treatment and the development of community hospital-based clinic models similar to RADIANS in the future. Further multicenter analyses will be warranted to determine whether the findings from this small retrospective study persist after undergoing more rigorous investigation.

\section{Funding}

T.M. received research funding from Novocure.

\section{Conflict of Interest}

None declared.

\section{References}

1 Potters L, Steinberg M, Rose C, et al; American Society for Therapeutic Radiology and Oncology; American College of Radiology. American Society for Therapeutic Radiology and Oncology and American College of Radiology practice guideline for the performance of stereotactic body radiation therapy. Int J Radiat Oncol Biol Phys 2004;60(4):1026-1032

2 McClelland S III, Kim E, Passias PG, Murphy JD, Attia A, Jaboin JJ. Spinal stereotactic body radiotherapy in the United States: a decade-long nationwide analysis of patient demographics, practice patterns, and trends over time. J Clin Neurosci 2017;46:109-112

3 Mahal BA, Chen YW, Sethi RV, et al. Travel distance and stereotactic body radiotherapy for localized prostate cancer. Cancer 2018;124(6):1141-1149

4 McClelland S III, Mitin T, Jaboin JJ, Ciporen JN. RADIANS: a multidisciplinary central nervous system clinic model for radiation oncology and neurosurgery practice. World Neurosurg 2019;122:8-10

5 Shen CJ, Hu C, Ladra MM, Narang AK, Pollack CE, Terezakis SA. Socioeconomic factors affect the selection of proton radiation therapy for children. Cancer 2017;123(20):4048-4056

6 Lee WR, Dignam JJ, Amin MB, et al. Randomized phase III noninferiority study comparing two radiotherapy fractionation schedules in patients with low-risk prostate cancer. J Clin Oncol 2016;34(20):2325-2332

7 Livi L, Meattini I, Marrazzo L, et al. Accelerated partial breast irradiation using intensity-modulated radiotherapy versus whole breast irradiation: 5-year survival analysis of a phase 3 randomised controlled trial. Eur J Cancer 2015;51(4):451-463 